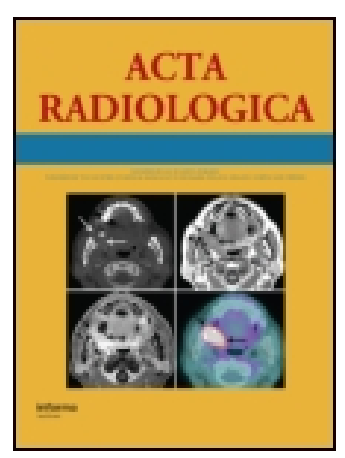

Acta Radiologica

ISSN: 0284-1851 (Print) 1600-0455 (Online) Journal homepage: https://www.tandfonline.com/loi/iard20

\title{
US and CT Findings in the Diagnosis of Tuberculous Peritonitis
}

\author{
F. B. Demirkazik, Okan Akhan, M. N. Özmen \& D. Akata
}

To cite this article: F. B. Demirkazik, Okan Akhan, M. N. Özmen \& D. Akata (1996) US and CT Findings in the Diagnosis of Tuberculous Peritonitis, Acta Radiologica, 37:4, 517-520

To link to this article: https://doi.org/10.3109/02841859609175435

册 Published online: 07 Jan 2010.

Submit your article to this journal

Џll Article views: 391

Q View related articles $\llbracket$

4 Citing articles: 2 View citing articles 


\title{
US AND CT FINDINGS IN THE DIAGNOSIS OF TUBERCULOUS PERITONITIS
}

\author{
F. B. Demirkazik, O. Akhan, M. N. Özmen and D. Akata \\ Department of Radiology, School of Medicine, Hacettepe University, Ankara, Turkey.
}

\begin{abstract}
Purpose: To evaluate the ultrasonographic and CT findings of tuberculous peritonitis.

Material and Methods: Ultrasonographic and CT findings of 11 patients with the ascitic type of tuberculous peritonitis were reviewed.

Results: All patients had ascites, and ultrasonography (US) demonstrated fine complete and incomplete mobile septations in 10 patients. In 5 of them, the ascites had a lattice-like appearance. Diffuse regular peritoneal thickening was detected in all patients by CT and in 10 patients by US. CT demonstrated infiltration of the greater omentum in 9 patients, whereas US showed omental thickening in only 5 patients.

Conclusion: Peritoneal and omental thickening detected by CT and ascites with fine, mobile septations shown by US strongly suggest the ascitic type of tuberculous peritonitis. The 2 imaging modalities should be used together for accurate diagnosis of tuberculous peritonitis.
\end{abstract}

Key words: Tuberculosis, peritoneal; ultrasonography; CT.

Correspondence: Okan Akhan, Radiology, Hacettepe Üniversitesi Tip Fakültesi, Sihhiye, Ankara, TR-06100, Turkey. FAX *90-312-31 12145.

Accepted for publication 14 August 1995.
Peritonitis is the most common manifestation of tuberculosis in the abdomen (15). Although sometimes asymptomatic, most of the patients have fever, weight loss, abdominal pain, and swelling (12). The diagnosis of tuberculous peritonitis (TBP) is difficult and sometimes delayed because of confusion of the disease with another illness and the nonspecificity of signs and symptoms (10).

Ascites is reported in $60-100 \%$ of cases (12). Therefore, TBP should be considered in the presence of ascites, especially in immunocompromised patients and in developing countries. Abdominal US is the first choice for investigation in such patients.

In this study of TBP, we report US and CT findings; such findings may lead to early diagnosis and treatment.

\section{Material and Methods}

Between 1990 and 1993, 11 patients with abdominal pain, abdominal swelling, fever, and malaise were diagnosed as having TBP. Six of the patients were male and 5 were female. Their ages ranged from 7 to 71 years with a mean of 34 years.

Peritoneal biopsy was performed in 6 patients, but only in 2 of them did the histopathologic examination reveal granulomatous infection. In the remaining 9 patients, the diagnosis was based on laboratory analyses of peritoneal fluid and improvement during antituberculous treatment.

All patients were examined with US and CT. US examinations were performed with a Toshiba SSA 90 A scanner equipped with $3.75-$ and $7.5-\mathrm{MHz}$ probes. Abdominal CT was obtained with a Philips Tomoscan 350 after administration of oral and i.v. contrast material using a slice thickness of 9 or 12 $\mathrm{mm}$. The US and CT features were assessed and classified according to a prospective protocol.

Five of the 11 patients ( $45 \%$ ) had unilateral pleural effusion, and one of these had also pericardial effusion. A 53-year-old man had active pulmonary tuberculosis and bilateral pleural effusion besides TBP. Another male patient with chronic renal failure had calcified pleural thickening suggestive of 
Table

US and CT features of 11 patients with TBP

\begin{tabular}{lrrrr}
\hline & \multicolumn{2}{c}{ US } & \multicolumn{2}{c}{ CT } \\
\cline { 2 - 5 } & $\mathrm{n}$ & $\%$ & $\mathrm{n}$ & $\%$ \\
\hline Ascites & 11 & 100 & 11 & 100 \\
Fine septations in the ascitic fluid & 10 & 91 & 4 & 36 \\
Lattice-like appearance of the & 5 & 45 & 0 & 0 \\
ascites & 10 & 91 & 11 & 100 \\
Peritoneal thickening & 6 & 55 & 9 & 82 \\
$\begin{array}{l}\text { Omental infiltration } \\
\text { Bowel wall and mesenteric }\end{array}$ & 10 & 91 & 11 & 100 \\
thickening & & & & \\
$\begin{array}{l}\text { Splenic involvement } \\
\text { Lymphadenopathy }\end{array}$ & 1 & 9 & 1 & 9 \\
& 1 & 9 & 1 & 9
\end{tabular}

healed tuberculous pleurisy. A 24-year-old female patient had chronic liver disease.

\section{Results}

The features of US and CT in TBP are summarized in the Table.

All patients had different amounts of ascites, and US showed fine complete and incomplete mobile strands of fibrin in the ascites in all except one (91\%) (Fig. 1). In 5 patients (45\%), the complete and incomplete septations had a lattice-like appearance (Fig. 2 a). CT showed fine septations in only 4 of 10 patients $(36 \%)$, especially in the sub-

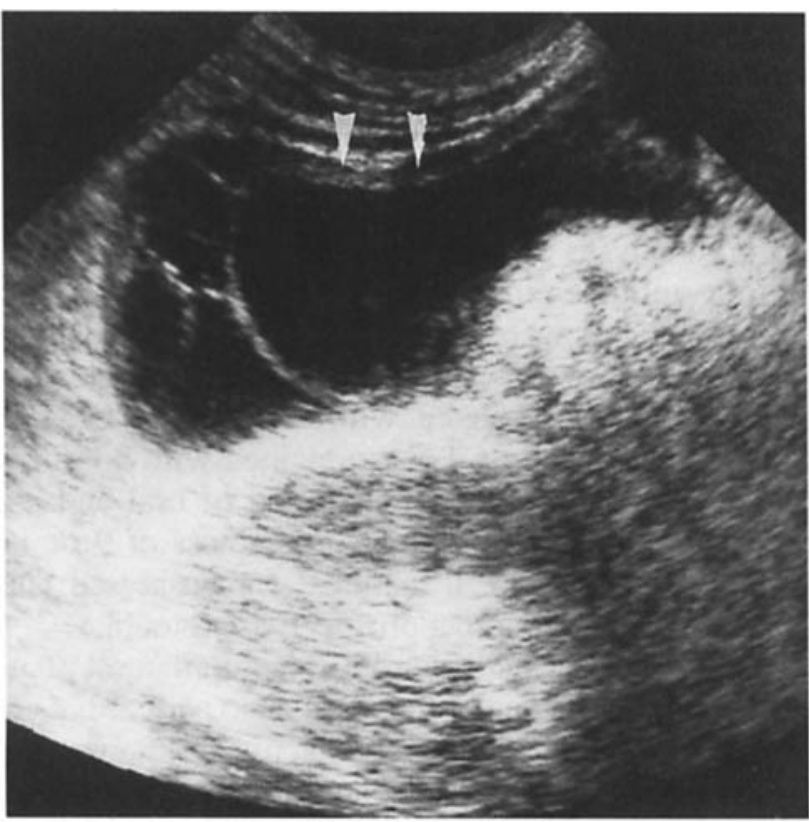

Fig. 1. Transverse US of the right paracolic gutter demonstrates fine septations in ascites and diffuse peritoneal thickening ( $>$ ). diaphragmatic and pelvic regions, and the septa were fewer than detected by US. Diffuse, regular peritoneal thickening of 2-6 $\mathrm{mm}$ was detected by US in 10 patients $(91 \%$ ) (Fig. 1), while peritoneal thickening and enhancement were demonstrated by $\mathrm{CT}$ in all patients (Fig. 2 b).

US failed to show peritoneal and bowel-wall thickening in a patient who had a small amount of ascites.

Peritoneal thickening was continuous with the mesentery and the small-bowel wall in all CT examinations (Figs 3 and 4). CT revealed different amounts of nodular infiltration of the greater omentum in 9 patients $(82 \%)$, sometimes forming a mass called "omental cake". US showed omental thickening in only 6 patients $(55 \%)$.

Both imaging modalities showed nodular infiltration of the spleen in one patient. Abdominal lymphadenopathy was detected in only one patient by US and CT.
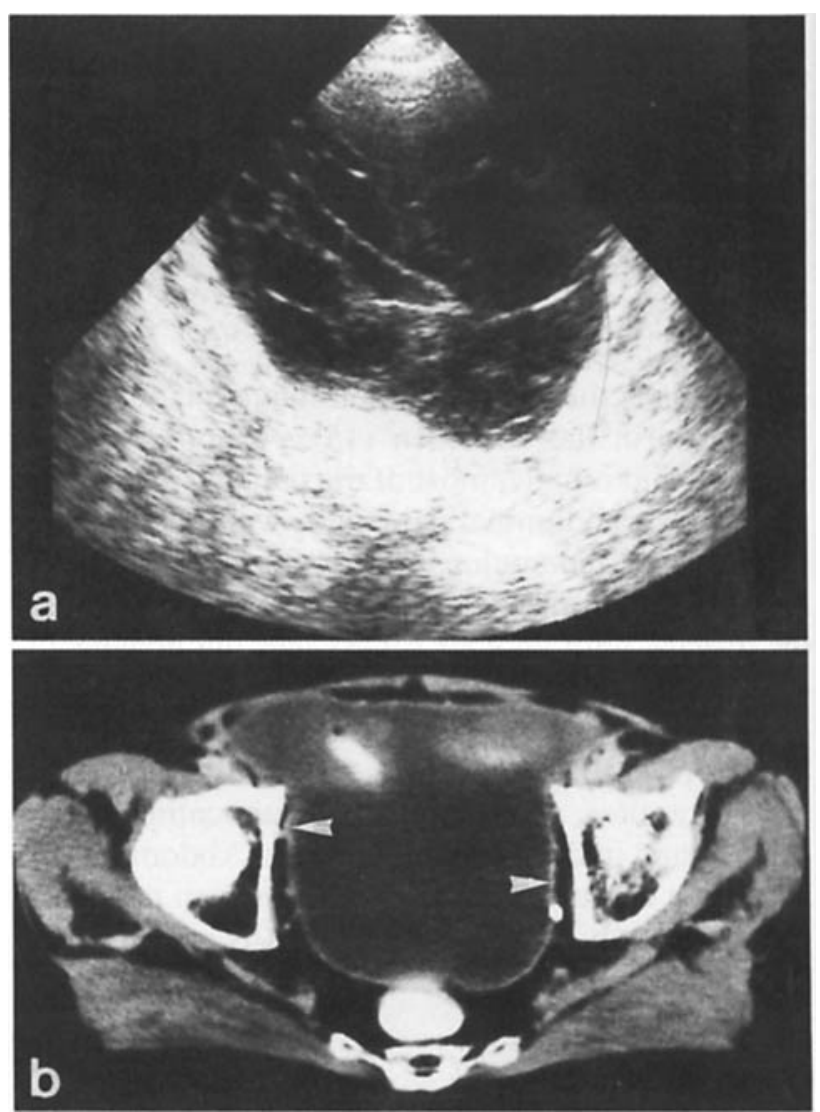

Fig. 2. a) Transverse US of the pelvic region shows complete and incomplete fine septations revealing a lattice-like appearance. b) CT of the same patient reveals ascites and diffuse peritoneal thickening with enhancement $(>)$. Note that no septation was demonstrated by CT. 


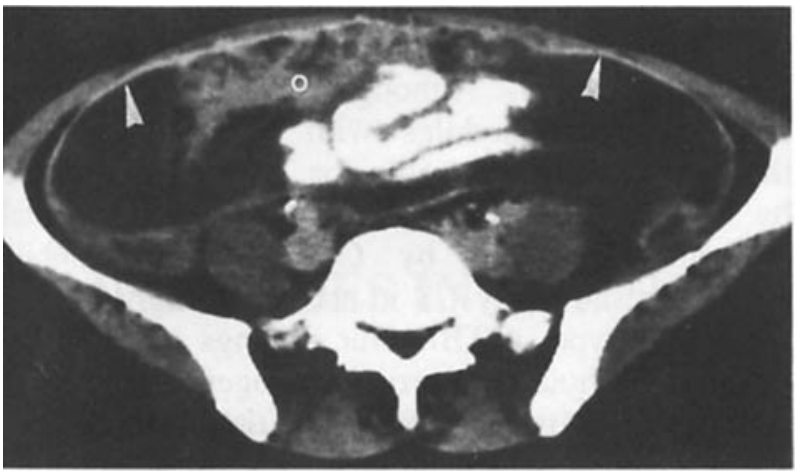

Fig. 3. CT of the lower abdomen demonstrates ascites, omental thickening (o), and peritoneal thickening with enhancement (>).

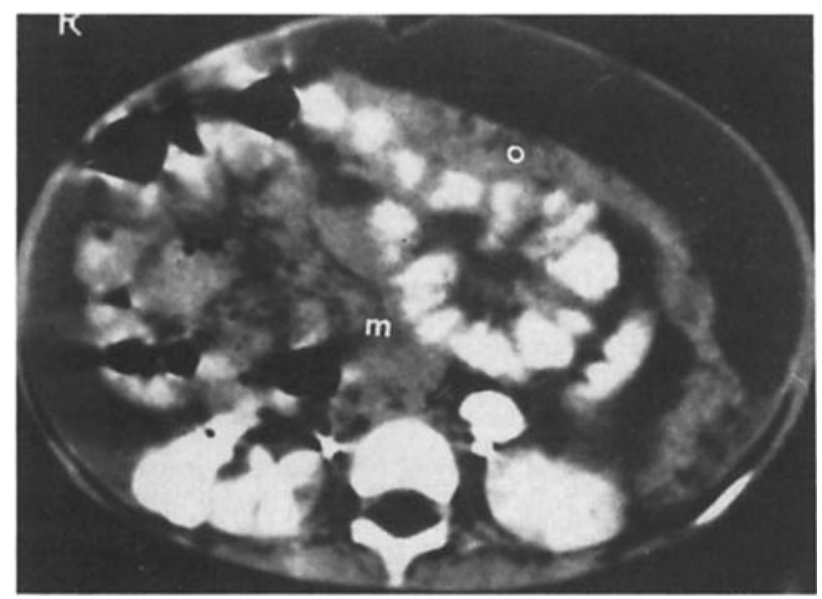

Fig. 4. CT of the middle abdomen shows ascites, thickening of the bowel wall, omental mass (o), and infiltration of the mesentery $(\mathrm{m})$.

\section{Discussion}

TBP is considered to develop as a result of hematogeneous dissemination from a distant focus or of spread by infection from diseased lymph nodes, the intestine, or the fallopian tubes (10).

Laparoscopic findings of TBP are free ascites and multiple nodules in the parietal and visceral peritoneum, most frequently associated with retraction and thickening of the greater omentum (9). In some patients, fibroadhesive types of TBP without ascites may be detected.

Barium studies may only demonstrate indirect signs of ascites or adhesions in TBP and, if present, signs of bowel involvement.

With the development of cross-sectional imaging modalities, more sensitive signs of TBP have been reported in some studies in which US and CT were used. Gompels \& Darlington (6) described fine, mobile septations forming a lattice-like structure in the ascites as sonographic features of TBP. ZIRINSKY et al. (17) reported loculated ascites containing multiple interlacing septations in one case. Another US feature of TBP was described as a "sliced bread" appearance of radially oriented strands of thickened small-bowel loops with a small amount of ascitic fluid (13). The first prospective study on US diagnosis of TBP was published by AKHAN et al. (1), who indicated the importance of numerous, thin, complete septations and fine, delicate, incomplete, mobile strands of deposited fibrin forming a lattice-like structure in the free ascites. The peritoneum was found to be thickened in their 4 patients. They found minute nodules on the surface of the parietal peritoneum. Similar findings have also been reported for the US diagnosis of tuberculous pleural effusions (2).

RamaiYa \& Walter (14) reported that most of their patients (20 of 31) had no US-detected septation, loculation, or echogenic material in the ascitic fluid. The results of their retrospective study conflict with the previous reports and our findings $(1,6,9)$. In our series, $91 \%$ of the patients had fine septations in the ascites, as detected by real-time US examination. The discrepancy between these studies may be explained by the difference of the methods applied. The study of Ramaiya \& Walter was a retrospective one, and US evaluation of the patients was done by reexamining the US reports and the films obtained previously (14).

In a retrospective study, fine septations, intestinal and peritoneal adhesions, and peritoneal thickening may be underdiagnosed because of insufficient attention during the routine abdominal US examination, as indicated also by RAMAIYA \& WALTER (14). We consider that thin septations in the ascites, bowel and peritoneal adhesions formed by these strands of fibrin, and peritoneal and omental thickening can be easily demonstrated by real-time US in a prospective study.

KEDAR et al. (11) stated that free or loculated ascites, lymphadenopathy, and peritoneal thickening were the predominant findings in the extraintestinal form of abdominal tuberculosis. Although they reported peritoneal thickening in 14 of 39 patients with ascites, they have not mentioned omental thickening. We detected omental thickening in 55\% of our cases by US and in $82 \%$ of them by CT.

An omental mass can be demonstrated by a meticulous US examination (16). When the omentum is infiltrated, it becomes a hyperechogenic, heterogeneous mass anterior to the bowel segments, and it may be difficult to distinguish this mass from the intestine and the anterior abdominal wall.

US can accurately demonstrate small quantities 
of ascitic fluid, as well as the delicate complete and incomplete mobile septa formations or internal echoes (1). In addition, intraperitoneal fluid facilitates the detection of peritoneal thickening down to $2 \mathrm{~mm}$ by separating the bowel loops and the parietal peritoneum.

Free and loculated ascites, thickening, nodularity, and enhancement of the peritoneum, mesentery, or omentum are the CT features of the ascitic type of $\operatorname{TBP}(4,5,7)$. Although our CT findings are generally consistent with previous reports, we have not detected any abdominal abscess in our patients. In our opinion, the thick solid rims surrounding low-density masses defined by EPSTEIN \& MANN (5) may represent enhancement by thickened peritoneum and loculated ascites rather than large caseous masses or an abscess.

In our study, all 11 patients had the ascitic type of TBP proved by biopsy and/or clinical response to antituberculous therapy. This is the first study on a series of TBP to compare US and CT findings. US is superior to $\mathrm{CT}$ in revealing fine complete and incomplete septations and internal echoes, whereas CT can demonstrate peritoneal thickening, omental mass, and thickening of the bowel wall and the mesentery better than US.

All of our patients had thickening of the mesentery and the intestinal wall, and diffuse regular peritoneal thickening demonstrated by CT. All but one patient had fine septations in ascites shown by US. When one considers that TBP involves all peritoneal surfaces, these findings are not surprising and should be considered highly specific for TBP.

Abdominal lymphadenopathy was the most common finding in abdominal tuberculosis in a previous study (8). However, this feature was encountered in only one of our patients. In another patient, multiple small focal lesions were recognized in the spleen, an uncommon involvement in abdominal tuberculosis.

In the differential diagnosis of TBP, peritoneal carcinomatosis and mesothelioma should be considered. Ascites, peritoneal thickening, omental infiltration, and adhesions are also features of primary or secondary tumors of the peritoneum. In mesothelioma, the ascites is disproportionately minimal in relation to the degree of tumor dissemination (3). The adhesive bands between the parietal peritoneum and intestinal loops are often thick and rigid in peritoneal malignancies, unlike the fine, mobile, complete and incomplete septations in TBP $(1,3)$. Although delicate septations may also be present in pyogenic peritonitis and hemoperitoneum, the US appearance of the ascites fluid changes rapidly with time, unlike the ascites of peritoneal tuberculosis.

CT findings of diffuse, regular peritoneal thickening less than $1 \mathrm{~cm}$, with or without nodules, and absence of intra-abdominal mass other than omental thickening are useful features of TBP in the differential diagnosis. Although it is difficult to demonstrate, small nodules with regular peritoneal thickening may be specific for TBP when detected by US $(1,14)$. Therefore, peritoneal and omental thickening detected by CT and fine, mobile septations shown by US in ascites strongly suggest the ascitic type of TBP. Our findings indicate that US and CT should be carried out together for a more accurate diagnosis. However, the definitive diagnosis of TBP can be made only with a tissue or fluid sample and positive cultures.

\section{REFERENCES}

1. Akhan O., Demirkazik F. B., Demirkazik A. et al.: Tuberculous peritonitis; ultrasonic diagnosis. J. Clin. Ultrasound 18 (1990), 711.

2. Akhan O., Demirkazik F. B., Özmen M. N. et al.: Tuberculous pleural effusions. Ultrasonic diagnosis. J. Clin. Ultrasound 20 (1992), 461.

3. Akhan O., Kalyoncu F., ÖZmen M. N. et al.: Peritoneal mesothelioma. Sonographic findings in nine cases. Abdom. Imaging 18 (1993), 280.

4. Dahle D. H., Stanley R. T., Koehler R. E. \& Shin M. S.: Abdominal tuberculosis. CT findings. J. Comput. Assist. Tomogr. 8 (1984), 443.

5. EPSTEIN B. M. \& MANN J. H.: CT of abdominal tuberculosis. AJR 139 (1982), 861.

6. Gompels B. M. \& Darlington L. G.: US diagnosis of tuberculous peritonitis. Br. J. Radiol. 51 (1978), 1018.

7. Hanson R. D. \& Hunter T. B.: Tuberculous peritonitis. CT appearance. AJR 144 (1985), 931.

8. Hulnick D. H., Megibow A. J., Naidich D. P., Hilton S., Cho K. C. \& Balthazar E. J.: Abdominal tuberculosis. CT evaluation. Radiology 157 (1985), 199.

9. Jorge A. D.: Peritoneal tuberculosis. Endoscopy 16 (1984), 10.

10. Karney W. W., O'Donoghue J. M., Ostrow J. H., Holmes K. K. \& Beaty H. N.: The spectrum of tuberculous peritonitis. Chest 72 (1977), 310.

11. Kedar R. P., Shah P. P., Shvinde R. S. \& Malde H. M.: Sonographic findings in gastrointestinal and peritoneal tuberculosis. Clin. Radiol. 49 (1994), 24.

12. Martin R. E. \& Bradsher R. W.: Elusive diagnosis of tuberculous peritonitis. South. Med. J. 79 (1986), 1076.

13. ÖzKan K., GürSES N. \& Gürses N.: Ultrasonographic appearance of tuberculous peritonitis. J. Clin. Ultrasound 15 (1987), 350.

14. Ramaiya L. I. \& Walter D. F.; Sonographic features of tuberculous peritonitis. Abdom. Imaging 18 (1993), 23.

15. Reeder M. M. \& Palmer P. E. S.: Infections and infestations. In: Alimentary tract radiology, p. 1482. Edited by A. R. Margulis \& H. J. Burhenne. C. V. Mosby, St. Louis 1989.

16. Wu C. C., Chow K. S., Lu T. N. \& Huang F. T.: Sonographic features of tuberculous omental cakes in peritoneal tuberculosis. J. Clin. Ultrasound 16 (1988), 195.

17. Zirinsky K., AuH Y. H., Kneeland J. B. et al.: Computed tomography, sonography and MR imaging of abdominal tuberculosis. Case report. J. Comput. Assist. Tomogr. 9 (1985), 961. 